\title{
Myasthenic Crisis After Recurrent COVID-19 Infection
}

CPT Adam M. Spanier, MD, USA; and CPT James I. Gragg, DO, USA

\author{
A patient with myasthenia gravis who survived 2 COVID-19 infections required \\ plasmapheresis to recover from an acute crisis.
}

\begin{abstract}
Adam Spanier is a Resident, and James Gragg is a Faculty Member, both at Carl R. Darnall Army Medical Center in Fort Hood, Texas. Correspondence: Adam Spanier (adam.m.spanier@gmail.com)
\end{abstract}

Fed Pract. 2021;38(8). Published online August 17. doi:10.12788/fp.0166
C OVID-19 is still in the early stages of understanding, although it is known to be complicated by individual patient comorbidities. The management and treatment of COVID-19 continues to quickly evolve as more is discovered regarding the virus. Multiple treatments have been preliminarily tested and used under a Food and Drug Administration emergency use authorization (EUA) determination. The long-term success of these therapies, however, is yet to be determined. Additionally, if a patient has a second clinical presentation for COVID19 , it is not known whether this represents latency with subsequent reactivation from the previous infection or a second de novo infection. The uncertainty calls into question the duration of immunity, if any, following a primary infection.

COVID-19 management becomes more complicated when patients have complex medical conditions, such as myasthenia gravis (MG). This autoimmune neuromuscular disorder can present with varying weakness, and many patients are on immunomodulator medications. The weakness can worsen into a myasthenic crisis $(\mathrm{MC})$, resulting in profound weakness of the respiratory muscles. Therefore, patients with MG are at increased risk for COVID-19 and may have a more complicated course when infected.

Our patient with MG presented for severe COVID-19 symptoms twice and later developed MC. He received 2 treatment modalities available under an EUA (remdesivir and convalescent plasma) for COVID-19, resulting in symptom resolution and a negative polymerize chain reaction $(\mathrm{PCR})$ test result for the virus.
However, after receiving his typical maintenance therapy of IV immunoglobulin (IVIG) for his MG, he again developed symptoms consistent with COVID-19 and tested positive. After recovering from the second episode of COVID-19, the patient went into MC requiring plasmapheresis.

\section{CASE PRESENTATION}

A 56-year-old male, US Army veteran presented to Carl R. Darnall Army Medical Center emergency department (ED) 6 days after testing positive for COVID-19, with worsening sputum, cough, congestion, dyspnea, and fever. Due to his MG, the patient had a home oxygen monitor and reported that his oxygenation saturation dropped below $90 \%$ with minimal exertion. His medical history was significant for MG, status postthymectomy and radiation treatment, left hemidiaphragm paralysis secondary to phrenic nerve injury, and corticosteroid-induced insulin-dependent diabetes mellitus. His current home medications included pyridostigmine $60 \mathrm{mg}$ 3 times a day, mycophenolate (MMF) $1500 \mathrm{mg}$ twice daily, IV immunoglobulin (IVIG) every 3 weeks, insulin aspart up to $16 \mathrm{U}$ per meal, insulin glargine $30 \mathrm{U}$ twice a day, dulaglutide $0.75 \mathrm{mg}$ every week, and metformin $1000 \mathrm{mg}$ twice daily.

On initial examination, the patient's heart rate (HR) was 111 beats/min, respiratory rate (RR), 22 breaths/min, blood pressure (BP), 138/88 mm Hg, temperature, $100.9^{\circ} \mathrm{F}$, and his initial pulse oximetry, $91 \%$ on room air. On physical examination, the patient was tachypneic, though without other signs of respiratory distress. Lung auscultation revealed no adventitial lung sounds. His cardiac examination 
FIGURE 1 Chest X-ray First Presentation to Emergency Department

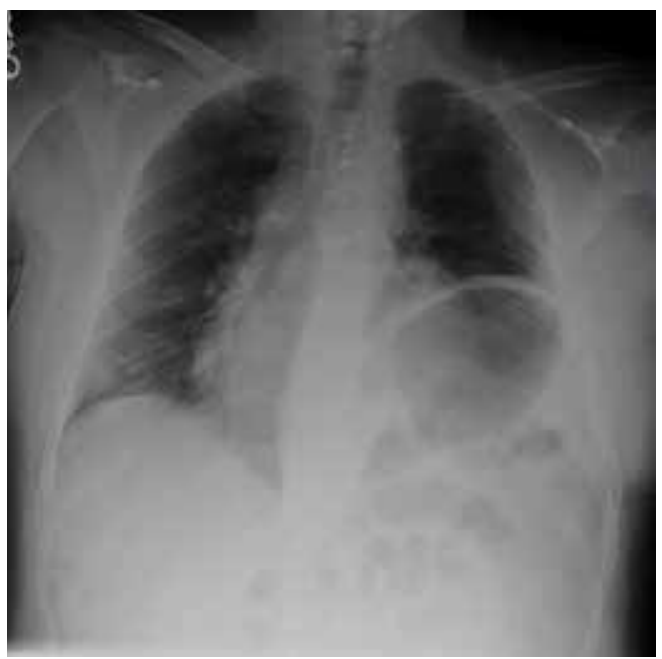

Notable for bibasilar opacities and patchy infiltrates with chronic left hemidiaphragm paralysis.

was notable only for tachycardia. His neurologic examination demonstrated intact cranial nerves, with 5 out of 5 (scale 1 to 5) strength throughout the upper and lower extremities, sensation was intact to light touch, and he had normal cerebellar function. The rest of the examination was normal.

Initial laboratory investigation was notable for a white blood cell count of $14.15 \times 10^{3}$ cells/mcL with $84 \%$ neutrophils, and 6\% lymphocytes. Additional tests revealed a C-reactive protein (CRP) level, $17.97 \mathrm{mg} / \mathrm{dL}$ (reference range, 0-0.5 $\mathrm{mg} / \mathrm{dL}$ ), ferritin level, $647 \mathrm{ng} / \mathrm{mL}$ (reference range, $22-274 \mathrm{ng} / \mathrm{mL}$ ), d-dimer, 0.64 $\mathrm{mcg} / \mathrm{mL}$ (reference range, $0-0.47 \mathrm{mcg} / \mathrm{mL}$ ), and a repeated positive COVID-19 PCR test. A portable chest X-ray showed bibasilar opacities (Figure 1).

The patient was diagnosed with COVID19 and admitted to the intensive care unit (ICU). In the ICU, the patient received $1 \mathrm{U}$ of convalescent plasma (CP) and started on a course of IV remdesivir $100 \mathrm{mg} / \mathrm{d}$ consistent with the EUA. He also received a 5-day course of ceftriaxone and azithromycin for possible community acquired pneumonia (CAP). As part of the patient's MG maintenance medications, he received IVIG $4 \mathrm{~g}$ while in the ICU. Throughout his ICU stay, he required supplemental nasal cannula oxygenation
FIGURE 2 Chest X-ray First Hospital Discharge

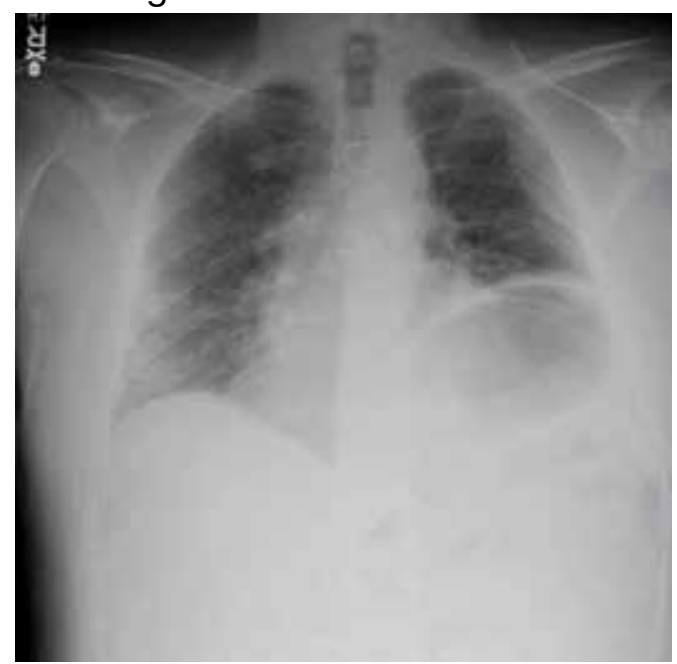

Improved opacities and stable chronic left hemidiaphragm has been seen.

to maintain his oxygen saturation $>93 \%$. After 8 days in the ICU, his oxygen requirements decreased, and the patient was transferred out of the ICU and remdesivir was discontinued. On hospital day 10, a repeat COVID-19 PCR test was negative, inflammatory markers returned to within normal limits, and a repeat chest X-ray showed improvement from admission (Figure 2). Having recovered significantly, he was discharged home.

Three weeks later, the patient again presented to the MTF with 3 days of dyspnea, cough, fever, nausea, and vomiting. One day before symptom onset, he had received his maintenance IVIG infusion. The patient reported that his home oxygen saturation was $82 \%$ with minimal exertion. On ED presentation his HR was 107 beats/min, RR, 28 breaths/min, temperature, $98.1^{\circ} \mathrm{F}$, BP $118 / 71 \mathrm{~mm} \mathrm{Hg}$, and oxygen saturation, $92 \%$ on $2 \mathrm{~L}$ nasal cannula. His examination was most notable for tachypnea with accessory muscle use. At this time, his neurologic examination was unchanged from prior admission with grossly intact cranial nerves and symmetric 5 of 5 motor strength in all extremities.

At this second ED visit, laboratory results demonstrated a CRP of $3.44 \mathrm{mg} / \mathrm{dL}$, ferritin $2019 \mathrm{ng} / \mathrm{mL}$, d-dimer, $3.39 \mathrm{mcg} / \mathrm{mL}$, and a positive COVID-19 PCR result. His chest X-ray demonstrated new peripheral 
opacities compared with the X-ray at discharge (Figure 3). He required ICU admission again for his COVID-19 symptoms.

During his ICU course he continued to require supplemental oxygen by nasal cannula, though never required intubation. This second admission, he was again treated empirically for CAP with levofloxacin $750 \mathrm{mg}$ daily for 5 days. He was discharged after 14 days with symptom resolution and down trending of inflammatory markers, though he was not retested for COVID-19.

Four days after his second discharge, he presented to the ED for a third time with diffuse weakness, dysphagia, and dysarthria of 1 day. His HR was 87/beats/min; $\mathrm{RR}, 17$ breaths/min; temperature, $98.7^{\circ} \mathrm{F}$; $\mathrm{BP}, 144 / 81 \mathrm{~mm} \mathrm{Hg}$; and oxygen saturation, $98 \%$ on room air. His examination was significant for slurred speech, bilateral ptosis, 3 of 5 strength in bilateral finger flexion/ abduction, wrist extension, knee and ankle flexion/extension; 4 of 5 strength in bilateral proximal muscle testing of deltoid, and hip; normal sensation, cerebellar function and reflexes. His negative inspiratory force (NIF) maximal effort was $-30 \mathrm{cmH}_{2} \mathrm{O}$. He was determined to be in MC without evidence of COIVD-19 symptoms, and laboratory results were within normal limits, including a negative COVID-19 PCR. As he received IVIG as maintenance therapy, plasmapheresis was recommended to treat his MC, which required transfer to an outside civilian facility.

At the outside hospital, the patient underwent 5 rounds of plasmapheresis over 10 days. By the third treatment his strength had returned with resolution of the bulbar symptoms and no supplemental oxygen requirements. The patient was discharged and continued his original dosages of MMF and pyridostigmine. At 3 months, he remained asymptomatic from a COVID-19 standpoint and stable from a MG standpoint.

\section{DISCUSSION}

Reinfection with the COVID-19 has been continuously debated with alternative explanations suggested for a positive test after a previous negative PCR test in the setting of symptom resolution. ${ }^{1,2}$ Proposed causes include dynamic PCR results due to prolonged viral shedding and inaccurate or poorly sensitive tests. The repeat positive cases in these scenarios, however, occurred in asymptomatic patients. ${ }^{1,2}$ COVID-19 shedding averages 20 to 22 days after symptom onset but has been seen up to 36 days after symptom resolution. ${ }^{2,3}$ This would suggest that fluctuating results during the immediate post symptom period may be due to variations in viral shedding load and or sampling errorespecially in asymptomatic patients. On the other hand, patients who experience return of symptoms days to weeks after previous convalescence leave clinicians wondering whether this represents clinical latency with reactivation or COVID-19 reinfection. A separate case of initial COVID-19 in a patient that had subsequent clinical recovery with a negative PCR developed recurrent respiratory symptoms and had a positive PCR test only 10 days later, further highlighting the reinfection vs reactivation issue of COVID-19. ${ }^{2}$ Further understanding of this issue may have implications on the extent of natural immunity following primary infection; potential vaccine dosage schedules; and global public health policies.

Although reactivation may be plausible given his immunomodulatory therapy, our patient's second COVID-19 symptoms started 40 days after the initial symptoms, and 26 days after the initial course resolution; previous cases of return of severe symptoms occurred between 3 and 6 days. ${ }^{1}$ Given our patient's time course between resolution and return of symptoms, if latency is the mechanism at play, this case demonstrates an exceptionally longer latency period than the ones that have been reported. Additionally, if latency is an issue in COVID-19, using remdesivir as a treatment further complicates the understanding of this disease.

Remdesivir, a nucleoside analogue antiviral, was shown to benefit recovery in patients with severe symptoms in the Adaptive COVID-19 Treatment Trial-1 study. ${ }^{4}$ Our patient had originally been placed on a 10-day course; however, on treatment day 8 , his symptoms resolved and the remdesivir was discontinued. This is a similar finding to half the patients in the 10-day arm of the study by McCreary and colleagues. ${ }^{5}$ Although our patient was asymptomatic 4 weeks after the start of remdesivir, 


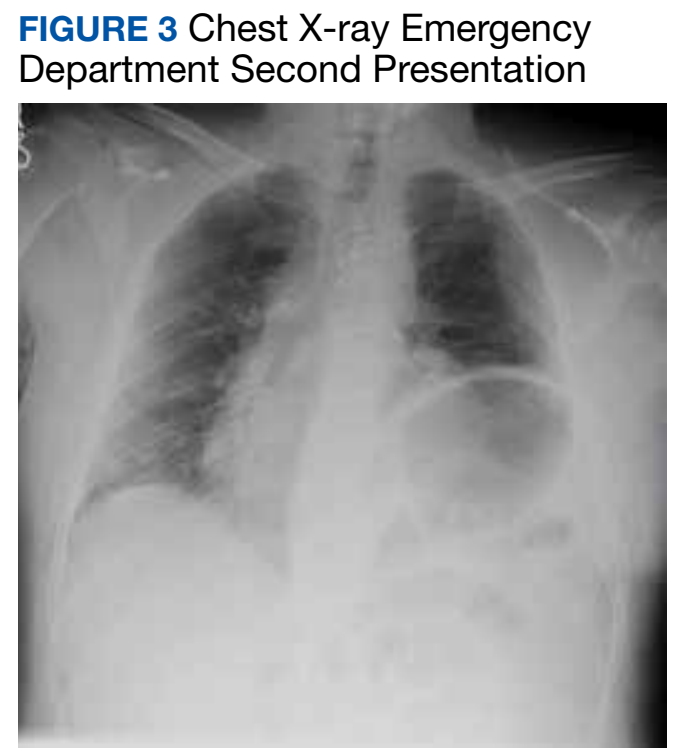

Notable for worsened bibasilar opacities and patchy infiltrates with stable chronic left hemidiaphragm paralysis.

consistent with the majority of patients in the McCreary 10-day study arm, further comparison of the presented patient is limited due to study length and follow-up considerations. ${ }^{5}$ No previous data exist on reactivation, reinfection, or long-term mortality after being treated with remdesivir for COVID-19 infection.

IVIG is being studied in the treatment of COVID-19 and bears consideration as it relates to our patient. There is no evidence that IVIG used in the treatment of autoimmune diseases increases the risk of infection compared with that of other medications used in the treatment of such diseases. Furthermore, the current guidance from the MG expert panel does not suggest that IVIG increases the risk of contracting COVID-19 aside from the risks of exposure to hospital infrastructure. ${ }^{6}$ Yet the guidance does not discuss the use of IVIG for MG in patients who are already symptomatic from COVID-19 or for patients recovering from the clinical disease or does it discuss a possible compounding risk of thromboembolic events associated with IVIG and COVID-19. ${ }^{6,7}$ Our patient received his maintenance IVIG during his first admission without any worsening of symptoms or increased oxygen requirements. The day following our patient's next scheduled IVIG infusion-while asymptomatic-he again developed respiratory symptoms; this could suggest that IVIG did not contribute to his second clinical course nor protect against.

$\mathrm{CP}$ is a treatment modality that has been used and studied in previous infectious outbreaks such as the first severe acute respiratory syndrome, and the H1N1 influenza virus. ${ }^{8}$ Current data on CP for COVID-19 are limited, but early descriptive studies have shown a benefit in improvement of symptoms 5 days sooner in those requiring supplemental oxygen, but no benefit for those requiring mechanical ventilation. ${ }^{9}$ Like patients that benefitted in these studies, our patient received CP early, 6 days after first testing positive and onset of symptoms. This patient's reinfection or return of symptoms draws into question the hindrance or even prevention of long-term immunity from administration of $C$.

COVID-19 presents many challenges when managing this patient's coexisting MG, especially as the patient was already being treated with immunosuppressing therapies. The guidance does recommend continuation of standard MG therapies during hospitalizations, including immunosuppression medications such as MMF. ${ }^{6}$ Immunosuppression is associated with worsened severity of COVID19 symptoms, although no relation exists to degree of immunosuppression and severity. ${ }^{7,10}$ To the best of our knowledge there has been no case report of reinfection or reactivation of COVID-19 associated with immunosuppressive agents used in the treatment of MG.

Our patient also was taking pyridostigmine for the treatment of his MG. There is no evidence this medication increases the risk of infection; but the cholinergic activity can increase bronchial secretions, which could theoretically worsen the COVID-19 respiratory symptoms. ${ }^{6,11}$ During both ICU admissions, our patient continued pyridostigmine use, observing complete return to baseline after discharge. Given the possible association with worsened respiratory outcomes after the second ICU admission, the balance between managing MG symptoms and COVID19 symptoms needs further examination.

The patient was in MC during his third presentation to the ED. Although respiratory symptoms may be difficult to differentiate from COVID-19, the additional neurologic symptoms seen in this patient allowed for 
quick determination of the need for MC treatment. There are many potential etiologies contributing to the development of the MC presented here, and it was likely due to multifactorial precipitants. A common cause of $M C$ is viral upper respiratory infections, further challenging the care of these patients during this pandemic. ${ }^{12}$ Many medications have been cited as causing a MC, 2 of which our patient received during admission for COVID-19: azithromycin and levoquin. ${ }^{12}$ Although the patient did not receive hydroxychloroquine, which was still being considered as an appropriate COVID19 treatment at the time, it also is a drug known for precipitating MC and its use scrutinized in patients with MG. ${ }^{12}$

A key aspect to diagnosing and guiding therapies in myasthenic crisis in addition to the clinical symptoms of acute weakness is respiratory assessment through the nonaerosolizing NIF test. ${ }^{12}$ Our patient's NIF measured $<30 \mathrm{cmH}_{2} \mathrm{O}$ when in $\mathrm{MC}$, while the reference range is $<75 \mathrm{cmH}_{2} \mathrm{O}$, and for mechanical ventilation is recommended at $20 \mathrm{cmH}_{2} \mathrm{O}$. Although the patient was maintaining $\mathrm{O}_{2}$ saturation $>95 \%$, his NIF value was concerning, and preparations were made in case of precipitous decline. Compounding the NIF assessment in this patient is his history of left phrenic nerve palsy. Without a documented baseline NIF, results were limited in determining his diaphragm strength. ${ }^{13}$ Treatment for MC includes IVIG or plasmapheresis, since this patient had failed his maintenance therapy IVIG, plasmapheresis was coordinated for definitive therapy.

\section{CONCLUSIONS}

Federal facilities have seen an increase in the amount of respiratory complaints over the past months. Although COVID-19 is a concerning diagnosis, it is crucial to consider comorbidities in the diagnostic workup of each, even with a previous recent diagnosis of COVID-19. As treatment recommendations for COVID-19 continue to fluctuate coupled with the limitations and difficulties associated with MG patients, so too treatment and evaluation must be carefully considered at each presentation.

\section{Author disclosures}

The authors report no actual or potential conflicts of interest with regard to this article.

\section{Disclaimer}

The opinions expressed herein are those of the authors and do not necessarily reflect those of Federal Practitioner, Frontline Medical Communications Inc., the US Government, or any of its agencies. This article may discuss unlabeled or investigational use of certain drugs. Please review the complete prescribing information for specific drugs or drug combinations-including indications, contraindications, warnings, and adverse effects - before administering pharmacologic therapy to patients.

\section{References}

1. Gousseff M, Penot P, Gallay L, et al. Clinical recurrences of COVID-19 symptoms after recovery: viral relapse, reinfection or inflammatory rebound? J Infect. 2020;81(5):816-846. doi:10.1016/j.jinf.2020.06.073

2. Duggan NM, Ludy SM, Shannon BC, Reisner AT, Wilcox SR. Is novel coronavirus 2019 reinfection possible? Interpreting dynamic SARS-CoV-2 test results. Am J Emerg Med. 2021;39:256.e1-256.e3. doi:10.1016/j.ajem.2020.06.079

3. Li J, Zhang L, Liu B, Song D. Case report: viral shedding for 60 days in a woman with COVID-19. Am J Trop Med Hyg. 2020;102(6):1210-1213. doi:10.4269/ajtmh.20-0275

4. Beigel JH, Tomashek KM, Dodd LE. Remdesivir for the treatment of Covid-19 - preliminary report. Reply. N Engl J Med. 2020;383(10):994. doi:10.1056/NEJMc2022236

5. McCreary EK, Angus DC. Efficacy of remdesivir in COVID-19. JAMA. 2020;324(11):1041-1042. doi:10.1001/jama.2020.16337

6. International MG/COVID-19 Working Group; Jacob S, Muppidi S, Gordon A, et al. Guidance for the management of myasthenia gravis (MG) and Lambert-Eaton myasthenic syndrome (LEMS) during the COVID-19 pandemic. J Neurol Sci. 2020;412:116803. doi:10.1016/j.jns.2020.116803

7. Anand P, Slama MCC, Kaku M, et al. COVID-19 in patients with myasthenia gravis. Muscle Nerve. 2020;62(2):254-258. doi:10.1002/mus.26918

8. Wooding DJ, Bach H. Treatment of COVID-19 with convalescent plasma: lessons from past coronavirus outbreaks. Clin Microbiol Infect. 2020;26(10):1436-1446. doi:10.1016/j.cmi.2020.08.005

9. Salazar E, Perez KK, Ashraf M, et al. Treatment of coronavirus disease 2019 (covid-19) patients with convalescent plasma. Am J Pathol. 2020;190(8):1680-1690. doi:10.1016/j.ajpath.2020.05.014

10. Ryan C, Minc A, Caceres J, et al. Predicting severe outcomes in Covid-19 related illness using only patient demographics, comorbidities and symptoms [published online ahead of print, 2020 Sep 9]. Am J Emerg Med. 2020;S07356757(20)30809-3. doi:10.1016/j.ajem.2020.09.017

11. Singh S, Govindarajan R. COVID-19 and generalized myasthenia gravis exacerbation: a case report. Clin Neurol Neurosurg. 2020;196:106045. doi:10.1016/j.clineuro.2020.106045

12. Wendell LC, Levine JM. Myasthenic crisis. Neurohospitalist. 2011;1(1):16-22. doi:10.1177/1941875210382918

13. Dubé BP, Dres M. Diaphragm dysfunction: diagnostic approaches and management strategies. $J$ Clin Med. 2016;5(12):113. Published 2016 Dec 5. doi: $10.3390 / j \mathrm{~cm} 5120113$ 